\title{
Thermodynamics of Ideal Pseudoelasticity
}

I. Müller

Technische Universität Berlin, Institut für Thermodynamik und Reaktionstechnik, 10623 Berlin, Germany

\begin{abstract}
Experimental results are shown for $\mathrm{CuZnAl}$ single crystals which summarize the thermal equation of state of these alloys in the pseudoelastic temperature range, i.e. their (P,d,T)behaviour. For a partial explanation the first and second law of thermodynamics are applied under the assumption that the essential relevant constitutive ingredients are

i.) a non-convex free energy

ii.) a coherency energy between the austenitic and martensitic regions.

The theory predicts an unstable phase equilibrium and allows the calculation of the heat emitted during the transition. It also determines which part of the heat is reversibly emitted and which irreversibly.
\end{abstract}

\section{PHENOMENA}

\section{$1.1(\mathrm{P}, \mathrm{d}, \mathrm{T})$-behaviour}

The load-deformation diagrams of a $\mathrm{CnZnAl}$ single crystal in the pseudoelastic temperature range are shown in Fig. 1. They are characterized by a hysteresis, and the interior of the hysteresis loop exhibits internal recovery (Fig. 1a), internal yield (Fig. 1b), internal loops (Fig. 1c) and internal elasticity (Fig. 1d). The plots of Fig. 1 through 4 and Fig. 6 were obtained by S. Fu and some of them were published in [1], [2]. Analogous phenomena occur in the deformation-temperature diagram and in the loaddeformation diagram; this is put in evidence in Fig. 2 and 3 respectively.

The $(\mathrm{P}, \mathrm{d})$-hystereses move upwards as temperature grows and the $(\mathrm{d}, \mathrm{T})$-hystereses move to the right, as shown in Fig. 4. Neither suffers a significant change of size.

\subsection{The diagonal}

It seems from Fig. 1 (in particular) that the hysteresis is traversed by a diagonal from the upper left to the lower right corner which, when reached from below or above, triggers internal yield and recovery respectively. The theoretical part of this paper in Section 2 will concentrate on a possible explanation of the diagonal as a line of unstable phase equilibrium.

It has recently been observed that the hysteresis at low temperature - which accompanies the twinning of the martensitic phase - also exhibits a diagonal with similar properties, see Fig. 5 . 

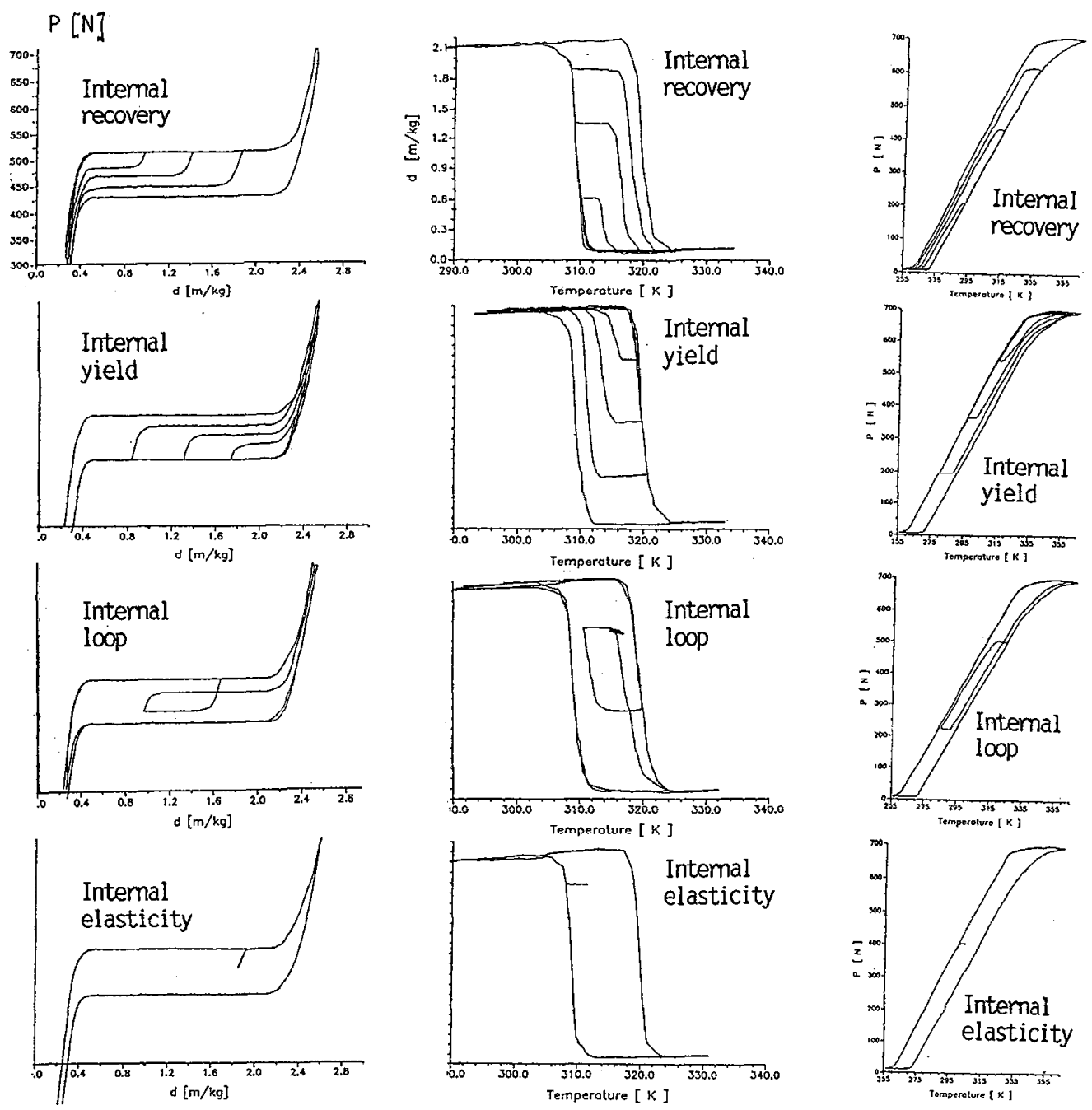

Fig. 1. (P,d)-diagrams

Fig. 2. (d,T)-diagrams

Fig. 3. (P,T)-diagrams
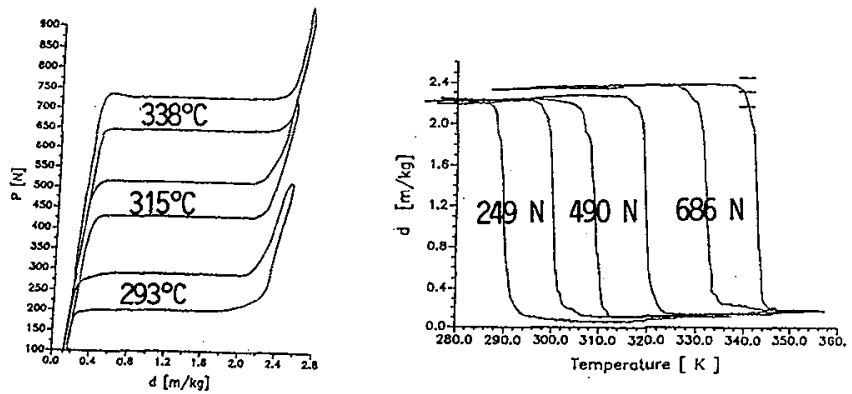

Fig. 4. Change of $(P, d)$-hysteresis with growing temperature Change of $(d, T)$-hysteresis with growing load. 


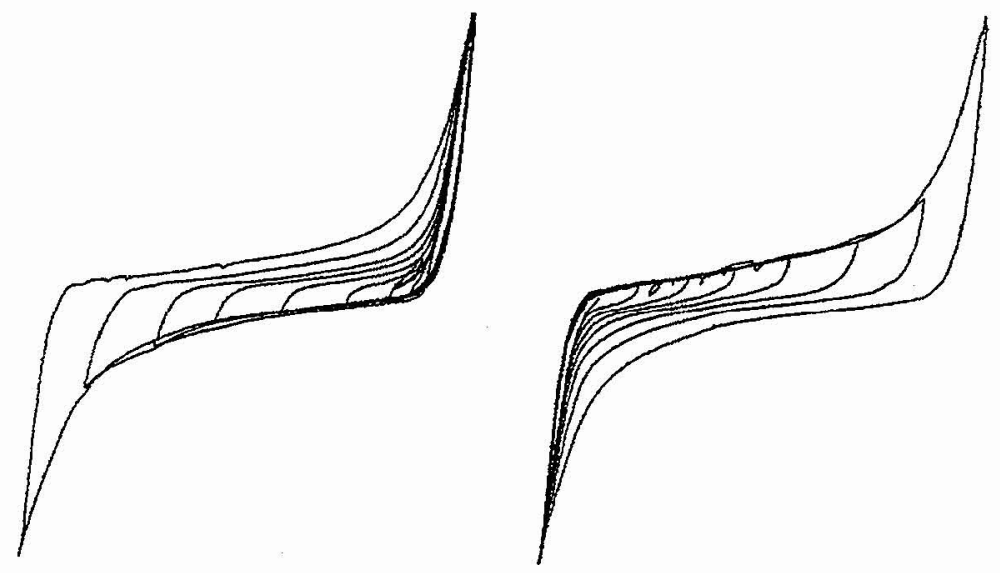

Fig. 5. Internal yield and recovery in martensitic twinning.

Courtesy V. Prieb [3]

\subsection{Relaxation and restitution}

If yield and recovery are interrupted and the deformation is kept constant for a long time in the points a through $f$ of Fig. $6 a$, we observe load relaxation or load restitution. These phenomena are not very pronounced, but they can clearly be seen, if the scale of the load axis is increased, see Fig. $6 \mathrm{~b}$ and Fig. $6 \mathrm{c}$.
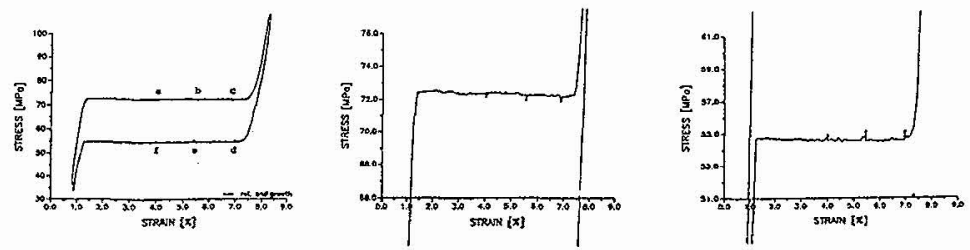

Fig. 6. Observed relaxation and restitution of load.

\subsection{Phase transition}

As the specimen moves along the yield line in the $(\mathrm{P}, \mathrm{d})$-diagram it undergoes a phase transition from the austenitic phase to a martensitic twin phase. The new phase appears in thin layers which increase in number - up to $40 / \mathrm{mm}$ at midpoint, see Fig. 7.
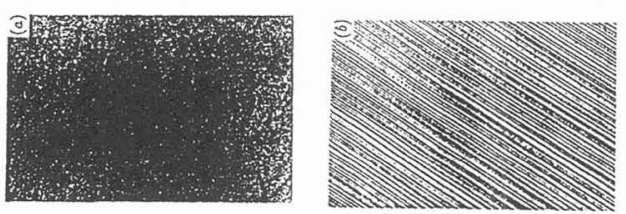

Fig. 7. Micrograph of the surface of the specimen

a.) $100 \%$ austenite, b.) $50 \%$ austenite and martensite. 


\section{THERMODYNAMICS}

\subsection{Phase Equilibrium}

\subsubsection{Stability Criteria}

The first and second law applied to a tensile specimen of length $\mathrm{D}$ under the Load $\mathrm{P}$ and with a uniform temperature $\mathrm{T}$ read

$$
\begin{aligned}
& \frac{\mathrm{dE}}{\mathrm{dt}}=\dot{Q}+\mathrm{P} \frac{\mathrm{dD}}{\mathrm{dt}} \Rightarrow \frac{\mathrm{d}(\mathrm{E}-\mathrm{TS})}{\mathrm{dt}}=\frac{\mathrm{dF}}{\mathrm{dt}}=-\mathrm{S} \frac{\mathrm{dT}}{\mathrm{dt}}+\mathrm{P} \frac{\mathrm{dD}}{\mathrm{dt}}-\mathrm{T} \Sigma \\
& \frac{\mathrm{dS}}{\mathrm{dt}}=\frac{\dot{\mathrm{Q}}}{\mathrm{T}}+\Sigma \quad \frac{\mathrm{d}(\mathrm{E}-\mathrm{TS}-\mathrm{PD})}{\mathrm{dt}}=\frac{\mathrm{dG}}{\mathrm{dt}}=-\mathrm{S} \frac{\mathrm{dT}}{\mathrm{dt}}-\mathrm{D} \frac{\mathrm{dP}}{\mathrm{dt}}-\mathrm{T} \Sigma
\end{aligned}
$$

$\Sigma$ is the non-negative entropy production. $F=E-T S$ and $G=E-T S-P D$ are the free energy and free enthalpy respectively. We conlude that

$\mathrm{F}$ tends to a mininum for $\mathrm{T}$ and $\mathrm{D}$ fixed

$\mathrm{G}$ tends to a minimum for $\mathrm{T}$ and $\mathrm{P}$ fixed.

\subsubsection{Yield, Recovery and Equilibrium Line}

Phase transitions require a non-convex free energy and - correspondingly - a non-monotone load deformation curve, see Fig. 8. In the present case the lower convex part corresponds to the austenitic phase, here denoted as phase', or phase". In a phase mixture the free energy $F$ and the length D of the specimen of mass $m$ is the sum of the free energies and lengths of the phases. We have

$$
\mathrm{F}=\mathrm{F}^{\prime}+\mathrm{F}^{\prime \prime}+\mathrm{Am} \mathrm{m}^{\prime} \frac{\mathrm{m}^{\prime \prime}}{\mathrm{m}} \quad \mathrm{D}=\mathrm{D}^{\prime}+\mathrm{D}^{\prime \prime}
$$
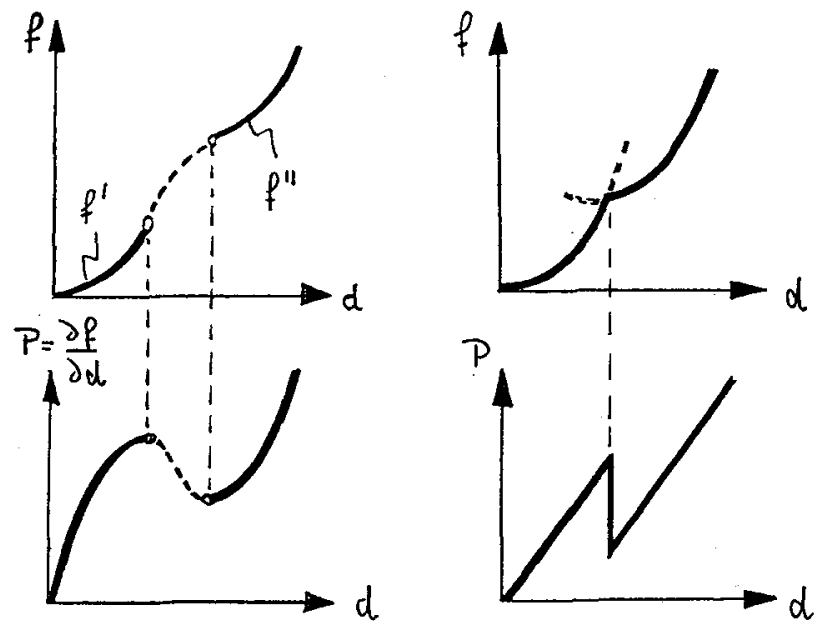

Fig. 8. Free energy and load vs. deformation Left: qualitative. Right: ideal.

In the free energy we have taken into account the coherency energy $A \mathrm{~m}^{\prime} \mathrm{m} " \mathrm{~m}$ which serves as an energetic penalty for the formation of interfaces. We assume - reasonably - that the number of interfaces is proportional to $\mathrm{m}^{\prime} \mathrm{m}^{\prime \prime} / \mathrm{m}$. The positive factor $\mathrm{A}$ is called the coherency factor. 
We divide the two equations (2.4) by $\mathrm{m}$ and obtain equations for an specific values, viz.

$$
\begin{aligned}
& f=(1-z) f^{\prime}\left(d^{\prime}, T\right)+z f^{\prime \prime}\left(d^{\prime \prime}, T\right)+A z(1-z) \\
& d=(1-z) \quad d^{\prime}+z \quad d^{\prime \prime} .
\end{aligned}
$$

For fixed temperature $f$ is a function of the three variables $d^{\prime}, d^{\prime \prime}$, and $z$, the phase fraction of martensite. In order to find the equilibrium values of these variables we minimize $f$ under the constraint of a fixed $d$. Or else we minimize

$$
\Phi\left(d^{\prime} d^{\prime \prime}, z, \lambda\right)=(1-z) f^{\prime}\left(d^{\prime}, T\right)+z f^{\prime \prime}\left(d^{\prime \prime}, T\right)+A z(1-z)-\lambda\left(d-(1-z) d^{\prime}-z d^{\prime \prime}\right)
$$

without constraint; $\lambda$ is a Lagrange multiplier. Necessary conditions for a minimum read

$$
\begin{aligned}
& (1-z)\left(\left.\frac{\partial \mathrm{f}^{\prime}}{\partial \mathrm{f}^{\prime}}\right|_{\mathrm{d}^{\prime}}+\lambda\right)=0, \\
& \left.\Rightarrow \frac{\partial \mathrm{f}^{\prime}}{\partial \mathrm{d}}\right|_{\mathrm{d}_{\mathrm{E}}^{\prime}}=\left.\frac{\partial \mathrm{f}^{\prime \prime}}{\partial \mathrm{d}}\right|_{\mathrm{d}_{\mathrm{E}}^{\prime \prime}}=-\lambda=\mathrm{P} \\
& \mathrm{z}\left(\left.\frac{\partial \mathrm{f}^{\prime \prime}}{\partial \mathrm{d}}\right|_{\mathrm{d}^{\prime \prime}}+\lambda\right)=0, \\
& -\lambda\left(\mathrm{d}_{\mathrm{E}}^{\prime \prime}-\mathrm{d}_{\mathrm{E}}^{\prime}\right)-\left(\mathrm{f}^{\prime \prime}\left(\mathrm{d}_{\mathrm{E}}^{\prime \prime}\right)\right)-\mathrm{f}^{\prime}\left(\mathrm{d}_{\mathrm{E}}^{\prime}\right)=\mathrm{A}\left(1-2 \mathrm{z}_{\mathrm{E}}\right)
\end{aligned}
$$

where the index $\mathrm{E}$ denotes equilibrium.

This equations (2.6) through (2.9) represent four equations for the four unknowns $d_{E}^{\prime}, d_{E}^{\prime \prime}, z_{E}$ and $\lambda$. As long as $f(d, T)$ is unspecified these equations are best solved graphically. We call $(2.7),(2.8)$ conditions of dynamical equilibrium because they ensure that the loads $\mathrm{P}=\frac{\partial \mathrm{f}}{\partial \mathrm{d}}$ are equal on the two phases and that the Lagrange multiplier $\lambda$ is equal to this (common) load except for sign. Equ. (2.9) is called condition of phase equilibrium; it may be written as

$$
P\left(d_{E}^{\prime \prime}-d_{E}^{\prime}\right)-\int_{d_{E}^{\prime}}^{d_{E}^{\prime \prime}} P(d) d d=A\left(1-2 z_{E}\right)
$$

The equation lends itself for an easy graphical construction: For a given $\mathrm{z}_{\mathrm{E}}$ we construct the rectangle $P\left(d_{E}^{\prime \prime}-d_{E}^{\prime}\right)$ which is bigger by $A\left(1-2 z_{E}\right)$ than the integral $\int P(d) d d$. Thus follows $d_{E}^{\prime}, d_{E}^{\prime \prime}, P$ and hence $d$ from (2.6). Fig. 9 shows this construction for $z_{E}=0,1$ and for an arbitrary $z_{E}$. The (P,d)-curve on which the phase equilibria lie has a negative slope which raises questions of stability which we shall discuss in the next section.

First, however, we conjecture that $\mathrm{P}^{(0)}$ in Fig $9 \mathrm{a}$ and $\mathrm{P}^{(1)}$ in Fig $9 \mathrm{~b}$ define the yield and recovery loads. This conjecture seems reasonable, since for these values of $P$ the phase equilibrium is established between the pure existing phase and the first trace of the emerging phase. Under that conjecture - as illustrated by Fig. 9 - the hysteresis loop rather obviously surrounds the area $2 \mathrm{~A}$.

Analogously we conjecture that the onset of internal yield and recovery occurs on the phase equilibrium line. This conjecture is supported by stability arguments, because we shall presently see that phase equilibrium is unstable. 

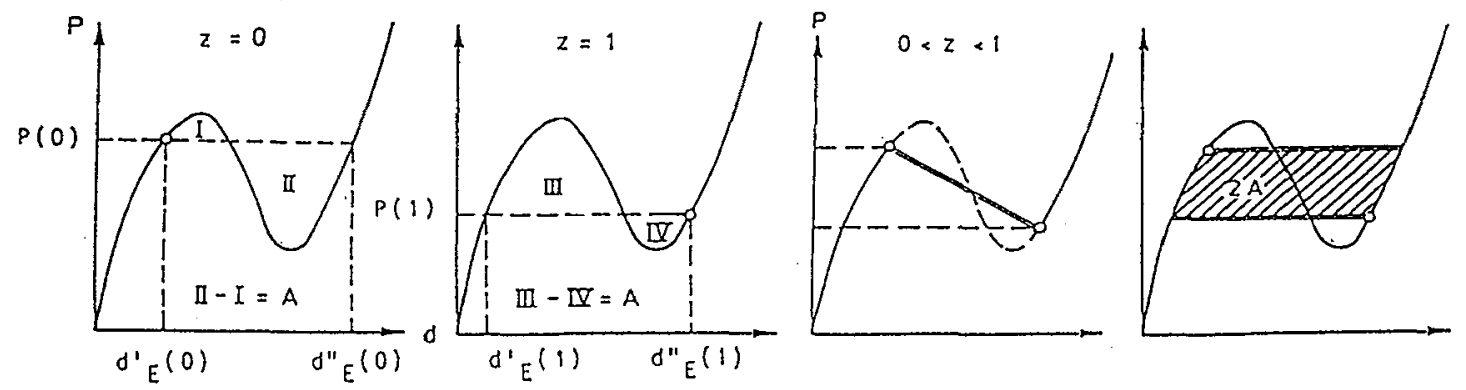

Fig. 9. Construction of $P, d^{\prime}, d^{\prime \prime}$ for a given $z$.

The size of the hysteresis loop.

\subsection{Ideal Pseudoelastictiy}

\subsubsection{Specific State Functions}

We consider the model of ideal pseudoelasticity in which both phases have the same elastic modulus $\alpha$ and the same constant specific heat $c$. In that case the specific free energies have the forms

$$
\begin{aligned}
& f^{\prime}(d, T)=\frac{1}{2} \alpha d^{2}+c\left(T-T_{R}\right)-c T \ln \frac{T}{T_{R}}+\varepsilon^{\prime}-T \eta^{\prime} \\
& f^{\prime \prime}(d, T)=\frac{1}{2}(d-\Delta)^{2}+c\left(T-T_{R}\right)-c T \ln \frac{T}{T_{R}}+\varepsilon^{\prime \prime}-T \eta^{\prime \prime}
\end{aligned}
$$

where $(d, T)=\left(0, T_{R}\right)$ is a reference state in which the internal energies and the entropies of the phases have the values $\varepsilon^{\prime}, \varepsilon^{\prime \prime}$ and $\eta^{\prime}, \eta^{\prime \prime}$. The free energy and the load of this model are shown on the R.H.S. of Fig. 8; they are given by a train of parabolae and straight lines respectively and $\Delta$ denotes the horizontal distance of the $(\mathrm{P}, \mathrm{d})$-curves of the phases and $\Delta_{\varepsilon}-T \Delta_{\eta}=\varepsilon^{\prime \prime}-\varepsilon^{\prime}-T\left(\eta^{\prime \prime}-\eta^{\prime}\right)$ denotes the vertical distance of the vertices of the parabolae. We take $\Delta_{\eta}$ to be negative.

The dynamic equilibrium conditions (2.7), (2.8) in this case read

$$
\alpha \mathrm{d}^{\prime}=\alpha\left(\mathrm{d}^{\prime \prime}-\Delta\right)=\mathrm{P} \quad \Rightarrow \quad \mathrm{d}^{\prime \prime}-\mathrm{d}^{\prime}=\Delta
$$

and the phase equilibrium condition (2.9) reads

$$
\mathrm{P} \Delta-\left(\Delta_{\varepsilon}-T \Delta_{\eta}\right)=\mathrm{A}\left(1-2 \mathrm{z}_{\mathrm{E}}\right) \quad \text { or, by (2.6) } \mathrm{P}=\frac{1}{1-\frac{2 \mathrm{~A}}{\alpha \Delta^{2}}}\left\langle-\frac{2 \mathrm{~A}}{\Delta^{2}} \mathrm{~d}+\frac{\Delta_{\varepsilon}-T \Delta_{\eta}+\mathrm{A}}{\Delta}\right\rangle \text {. }
$$

Equ. $(2.13)_{2}$ represents the analytic form of the downward sloping phase equilibrium line that was previously constructed graphically. In this model the line is straight. Since $\Delta_{\eta}<0$ holds, the line moves upwards as temperature grows, thus making the hysteresis in the (P,d)-diagram stand higher for higher temperature.

The (d,T)- and $(\mathrm{P}, \mathrm{T})$-diagrams each have their own phase equilibrium line which is also represented by the equation $(2.13)_{2}$. 


\subsubsection{Stability of Phase Equilibrium}

In order to simplify the stability argument we assume that the dynamic equilibrium conditions (2.12) are already statisfied, while the phase equilibrium is still in the process of being established. In this way the free energy $f$ and the free enthalpy $g=f-P d$ may be written as functions of $d, T, z$ and $P, T, z$ respectively. We obtain

$$
\begin{aligned}
\mathrm{f}(\mathrm{z} ; \mathrm{d}, \mathrm{T})= & \frac{\alpha}{2} \mathrm{~d}^{2}+\mathrm{c}\left(\mathrm{T}-\mathrm{T}_{\mathrm{R}}\right)-\mathrm{cT} \ln \frac{\mathrm{R}}{\mathrm{T}_{\mathrm{R}}}+\varepsilon^{\prime}-\mathrm{T} \eta^{\prime}+ \\
& +\mathrm{z}\left[-\alpha \Delta \mathrm{d}+\Delta_{\varepsilon}-\mathrm{T} \Delta_{\eta}+\mathrm{A}\right]+\mathrm{z}^{2}\left[\frac{\alpha}{2} \Delta^{2}-\mathrm{A}\right] \\
\mathrm{g}(\mathrm{z} ; \mathrm{P}, \mathrm{T})= & -\frac{\mathrm{P}^{2}}{2 \alpha}+\mathrm{c}\left(\mathrm{T}-\mathrm{T}_{\mathrm{R}}\right)-\mathrm{cT} \ln \frac{\mathrm{T}}{\mathrm{T}_{\mathrm{R}}}+\varepsilon^{\prime}-\mathrm{T}^{\prime}+ \\
& +\mathrm{z}\left[-\mathrm{P} \Delta+\Delta_{\varepsilon}-\mathrm{T} \Delta_{\eta}+\mathrm{A}\right]+\mathrm{z}^{2} \quad[-\mathrm{A}]
\end{aligned}
$$

Thus for fixed d,T the free energy is a function of $\mathrm{z}$ which - by $(2.3)_{1}$ - must be minimal in equilibrium. This condition reads

$$
\frac{\partial^{2} \mathrm{f}}{\partial \mathrm{z}^{2}}=\frac{\alpha}{2} \Delta^{2}-\mathrm{A}>0
$$

Also for fixed $P, T$ the free enthalpy as a function of $z$ must have a minimum for the equilibrium to be stable. Thus

$$
\frac{\partial^{2} g}{\partial z^{2}}=-A>0
$$

Since $A$ is positive - albeit small compared to elastic energies - the first condition is satisfied but the second condition is not. Therefore we conclude that the points on the phase equilibrium line are akin to saddle points, i.e. they are unstable under load-control.

While the whole specimen is ,length-controlled“ in the experiments of Chapter 1, the individual layers in the specimen feel the load needed to maintain the total length. We may say that the individual layers are load-controlled and therefore the phase equilibrium is unstable. This consideration supports the conjecture that internal yield and recovery is triggered on the phase equilibrium line.

\subsection{Entropy Production and Latent Heat}

\subsubsection{Entropy Production}

While it is interesting to investigate the equilibrium properties, the fact remains, that the specimen does not run through the equilibrium states. Rather it moves along the yield and recovery lines which are not equilibria and therefore this motion must create entropy. We proceed to calculate the entropy production.

We use (2.1) to calculate the specific entropy production $\sigma=\Sigma / \mathrm{m}$

$$
\begin{aligned}
& -\mathrm{T} \sigma=\frac{\mathrm{df}}{\mathrm{dt}}+\mathrm{s} \frac{\mathrm{dT}}{\mathrm{dt}}-\mathrm{P} \frac{\mathrm{dd}}{\mathrm{dt}} \quad \text { with }(2.5),(2.6) \\
& -\mathrm{T} \sigma=\underline{\left(\frac{\mathrm{df}}{\mathrm{dt}}+\mathrm{s}\right)} \frac{\mathrm{dT}}{\mathrm{dt}}+(1-\mathrm{z}) \underline{\left(\frac{\partial \mathrm{f}^{\prime}}{\partial \mathrm{d}^{\prime}}-\mathrm{P}\right)} \frac{\mathrm{dd^{ \prime }}}{\mathrm{dt}}+\mathrm{z}\left(\underline{\left.\frac{\partial \mathrm{f}^{\prime \prime}}{\partial \mathrm{d}^{\prime \prime}}-\mathrm{P}\right)} \frac{\mathrm{dd^{ \prime \prime }}}{\mathrm{dt}}-\left[\mathrm{P}\left(\mathrm{d}^{\prime \prime}-\mathrm{d}^{\prime}\right)-\int_{\mathrm{d}^{\prime}}^{\mathrm{d}^{\prime \prime}} \mathrm{Pdd}-\mathrm{A}(1-2 \mathrm{z})\right] \frac{\mathrm{dz}}{\mathrm{dt}} .\right.
\end{aligned}
$$


Assuming that in yield and recovery we have thermal and dynamic equilibrium the underlined terms are equal to zero and we have

$$
T \sigma=\left[P\left(d^{\prime \prime}-d^{\prime}\right)-\int_{d^{\prime}}^{d^{\prime \prime}} P d d-A(1-2 z)\right] \frac{d z}{d t} .
$$

We recall from Section 2.1.2. or 2.2.1. (Equ. (2.13) $)$

$$
P\left(d^{\prime \prime}-d^{\prime}\right)-\int_{d^{\prime}}^{d^{\prime \prime}} P d d=\left\{\begin{aligned}
A \\
-A
\end{aligned} \text { on the } \begin{array}{ll}
\text { yield } & \text { recovery }
\end{array}\right. \text { line }
$$

so that (2.19) reads

$$
T \sigma=\left\{\begin{array}{lll}
2 A z & \frac{d z}{d t} & \text { yield } \\
-2 A(1-z) & \frac{d z}{d t} & \text { recovery }
\end{array}\right.
$$

If we integrate from the beginning of yield or recovery at $z=0$ or $z=1$ respectively up to an arbitrary value of $z$ we obtain

$$
\int T \sigma d t= \begin{cases}A z^{2} & \text { yield } \\ A(z-1)^{2} & \text { re covery }\end{cases}
$$

Therefore the entropy production proceeds quadratically with the change of $z$.

\subsubsection{Latent Heat}

The specific heating $\dot{q}=\dot{Q} / \mathrm{m}$ during the process of isothermal yield and recovery follows from (2.2)

$$
\dot{\mathrm{q}}=\mathrm{T} \frac{\mathrm{d} \mathbf{s}}{\mathrm{dt}}-\mathrm{T} \sigma
$$

Since $s=-\frac{\partial f}{\partial T}$ holds in thermal equilibrium, we have for an ideally pseudoelastic body

$$
\dot{\mathrm{q}}=\mathrm{T} \Delta_{\eta} \frac{\mathrm{dz}}{\mathrm{dt}}-\left\{\begin{array}{lll}
2 \mathrm{Az} & \frac{\mathrm{dz}}{\mathrm{dt}} & \text { yield } \\
-2 \mathrm{~A}(1-\mathrm{z}) & \frac{\mathrm{dz}}{\mathrm{dt}} & \text { recovery }
\end{array}\right.
$$

Integration starting from $z=0$ and $z=1$ respectively yields

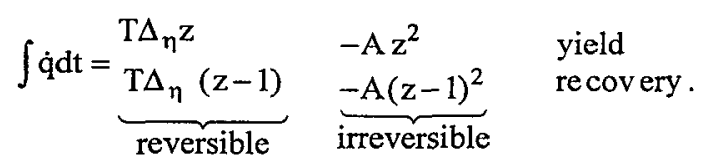

We recall that $\Delta_{\eta}<0$ holds and conclude that the latent heat has reversible and irreversible parts as indicated in (2.22). The reversible part is negative during yield and positive during recovery, while the irreversible part is always negative. 
The total heating in a full cycle of yield and recovery is given as

$$
\mathrm{q}_{0}=-2 \mathrm{~A}
$$

which corresponds to the area of the hysteresis loop - as it should -, see Paragraph 2.1.2.

\section{References}

[1] Fu, S., Huo, Y., Müller, I., Thermodynamics of pseudoelasticity - an analytical approach, Acta Mechanica 99 (1993) 1-19.

[2] Huo, Y., Müller, I., Non-equilibrium thermodynamics of pseudoelasticity, Cont. Mech. Thermodyn. 5 (1993) 163-204.

[3] Prieb, V., Hystereseverhalten und Thermoelastizität von Gedächtnislegierungen, private communication (1994).

Further relevant references

\section{On pseudoelasticity}

Lü, L., Aernouldt, E., Delay, L., Hysteresis effects of martensitic transformations during thermodynamical cycling. Scr. metall. 22 (1988) 1435-1440.

Müller, I., Xu, H., On the pseudoelastic hysteresis. Acta Metall. 39 (1991) 263-271.

\section{On thermodynamics}

Ortín, J., Planes, A., Thermodynamics of thermoelastic martensitic transformations. Acta Metall. 37 (1989) 1433-1441 and 1873-1881.

Huo, Y., Müller, I., Thermodynamics of pseudoelasticity - a graphical approach. In: Models of hysteresis (ed. A. Visintin), Pitman Research Notes in Mathematics 286 (1993) 39-58. 